\title{
Mechanical Properties and Water Permeability of A-B-A Tri-Block Copolymer Membranes Consisting of Poly( $\gamma$-benzyl L-glutamate) as the A-Component and Polybutadiene as the B-Component
}

\author{
Akio NaKaJIMA, Kouhei KugO, and Toshio HAYASHI \\ Department of Polymer Chemistry, Kyoto University, \\ Sakyo-ku, Kyoto 606, Japan.
}

(Received August 13, 1979)

\begin{abstract}
The mechanical properties of and the hydraulic permeability of water toward membranes of A-B-A tri-block copolymers consisting of $\alpha$-helical poly ( $\gamma$-benzyl L-glutamate) as the A-component and polybutadiene as the B-component, were examined and compared with the same properties of poly( $\gamma$-benzyl L-glutamate) membrane. Analysis of the temperature dependence of the dynamic modulus and the loss modulus based on Takayanagi's mechanical model leads to the conclusion that the dynamic mechanical spectra can be well explained by the micro-heterophase structure observed by electron microscope. The hydraulic permeability $K$ of water for the block copolymer membranes is dramatically higher than that of homopolypeptide, and increases in proportion to the interfacial area between the A and B domains. This fact suggests that the residues near the end of polypeptide chain and the terminal residues of amine-terminated polybutadiene, which locate in the interfacial region between the $\alpha$-helical A-component and the B-component, are responsible for the water permeability, since $\mathrm{NH}$ and $\mathrm{CO}$ residues in this region do not form intramolecular hydrogen bonds.
\end{abstract}

KEY WORDS Tri-Block Copolymer / Poly $(\gamma$-benzyl L-glutamate) / Polybutadiene / Mechanical Properties / Water Permeability /

In preceding papers, ${ }^{1.2}$ we investigated the formation and structure of micro-heterophase for A-B-A tri-block copolymers consisting of $\alpha$-helical poly ( $\gamma$-benzyl L-glutamate) as the A-component and polybutadiene as the B-component, and showed a novel micro-heterophase structure in which the polypeptide chains in $\alpha$-helical rod conformation aggregate in the A-domains, and polybutadiene chains in Gaussian coil conformation aggregate in the B-domains. The shape and size of domains evaluated from thermodynamic considerations on micelle formation were in good agreement with those obtained from the electron micrograph for cast membranes. It was also pointed out that the interfacial free energy between A and B domains per unit area played an important role in the micelle formation. The larger the interfacial free energy, the more repulsive $\mathrm{A}$ and $\mathrm{B}$ components; i.e., the more preferable micro-phase separation does occur.

Dynamic mechanical spectroscopy has been used for characterizing multi-component polymer systems, such as block and graft copolymers, polymer blends, and filled polymers. The dynamic mechanical relaxation behavior and the tensile properties of the present block copolymer membranes exhibiting a novel heterophase structure will be discussed first. In particular, the dynamic mechanical spectra for the block copolymer membranes can be visualized by applying the equivalent mechanical model as proposed by Takayanagi, ${ }^{3}$ for ascertaining consistency in the heterophase structure pointed in our previous paper. $^{2}$

The later portion of this paper concerns the permeability properties of the membranes. The polybutadiene portion of the block copolymers is fully hydrophobic so that the B-domain is controlled only by dispersion force. Contrarily, poly $(\gamma$-benzyl Lglutamate) chain portion includes $\mathrm{NH}$ and $\mathrm{CO}$ residues, hence, the A-domain is governed not only by dispersion force but also by polar and hydrogenbonding forces, as was indicated with respect to the surface tension of component blocks. ${ }^{2}$ It is known that at least four peptide residues at the $\mathrm{C}$-terminal of a peptide chain do not participate in the $\alpha$-helix in 
a helicogenic solvent. Also, the C-terminal of the present polypeptide is connected with polybutadiene chain end via $-\mathrm{X}-\mathrm{NH}-\mathrm{CO}$ - residue, where $\mathrm{X}$ is a residue including some $\mathrm{C}$ and $\mathrm{N}$ atoms (see below). Accordingly, the chain portion near the end of Acomponent connected with B-component chain has a random coil conformation consisting of several residues. The water permeability of the block copolymer membranes cast from chloroform will be discussed by considering the behavior of such interfacial regions toward water.

\section{EXPERIMENTAL}

\section{Materials}

A cyclo-aliphatic secondary amine-terminated polybutadiene (kindly supplied by Drs. K. Rieu and R. Drake of the B. F. Goodrich Chem. Co.) having a number-average molecular weight of 3,600 (degree of polymerization of 64) was used as the middle block. The molecular weight distribution of this block was very sharp. The A-B-A tri-block copolymers were prepared by reacting the middle block with $N$-carboxy anhydride of $\gamma$-benzyl L-glutamate in the absence of moisture at room temperature in a dioxane-methylene dichloride mixture. Following polymerization, the copolymers were precipitated in methanol for purification, and then dried in vacuo. The A-B-A tri-block copolymer obtained is in the form of

$$
\begin{aligned}
& \mathrm{H}-(\mathrm{NH}-\mathrm{CHR}-\mathrm{CO})_{n}-\mathrm{X}-\mathrm{NH}-\mathrm{CO}- \\
& \left(\mathrm{CH}_{2}-\mathrm{CH}=\mathrm{CH}-\mathrm{CH}_{2}\right)_{m}-\mathrm{CO}-\mathrm{NH}-\mathrm{X}- \\
& \quad(\mathrm{CO}-\mathrm{CHR}-\mathrm{NH})_{n}-\mathrm{H}
\end{aligned}
$$

where $\mathrm{R}$ and $\mathrm{X}$ denote $-\left(\mathrm{CH}_{2}\right)_{2}-\mathrm{COOCH}_{2}-$ and $-\sqrt{\mathrm{N}} \mathrm{N}-\mathrm{CH}_{2}-\mathrm{CH}_{2}-$, respectively.
The copolymer composition was determined by ultraviolet spectra and elemental analysis. Since $P_{\mathrm{B}}(=64)$ is known, the degrees of polymerization of the A-block, $P_{\mathrm{A}}$ was estimated from the copolymer composition. In Table I, the copolymer composition, denoted by $\mathrm{mol}^{\%} \%$ of the component A, the helix content $X_{\mathrm{H}}$ of copolymers in solution, and the interfacial area $\Gamma_{\mathrm{c}}$ between the A and B domains per unit volume micelle, estimated in a preceding paper, ${ }^{2}$ are summarized together with $P_{\mathrm{A}}$.

\section{Membrane Preparation}

To study mechanical properties and permeability, membranes of 10 to $50 \mu \mathrm{m}$ in thickness were cast onto glass plates from about $2 \%$ solutions in chloroform. The residual solvent in the air-dried membranes was removed by methanol and ether. The membranes were then dried in vacuo for 3 days at room temperature prior to the measurements.

\section{Mechanical Property Measurements}

The dynamic mechanical relaxation behavior was measured with a DDV-II Rheovibron at a frequency of $110 \mathrm{~Hz}$ and a heating rate of $0.3^{\circ} \mathrm{C} / \mathrm{min}$ over a temperature ranging from -20 to $100^{\circ} \mathrm{C}$. The tensile properties were measured on a Tensilon UTM-II-20 (Toyo-Boldwin Co.) using dog-bone samples of $1.0 \times 0.4 \mathrm{~cm}$ with thicknesses of 30 to $50 \mu \mathrm{m}$. All the samples were tested under an elongation rate of $40 \%$ per minute at $25^{\circ} \mathrm{C}$.

\section{Permeability Measurements}

The hydraulic permeability of the membranes under pressure was measured with a low-pressure ultrafiltration cell of Bio-Engineering Co. (Model MC-II). The exposed membrane area was $12.57 \mathrm{~cm}^{2}$. The cell was immersed in a water bath whose

Table I. Molecular and conformational parameters of GBG block copolymers and PBLG homopolymer

\begin{tabular}{lrrrrr}
\hline Code & $P_{\text {A }}$ & $P_{\mathbf{B}}$ & $\mathrm{A} / \mathrm{mol} \%$ & $X_{\mathrm{H}}$ & $\Gamma_{\mathrm{c}} \times 10^{3} / \AA^{-1}$ \\
\hline GBG-1 & 53 & 64 & 67.5 & 0.681 & 10.03 \\
GBG-2 & 78 & 64 & 71.6 & 0.719 & 8.41 \\
GBG-3 & 119 & 64 & 78.1 & 0.788 & 6.64 \\
GBG-4 & 188 & 64 & 81.3 & 0.812 & 4.91 \\
GBG-5 & 224 & 64 & 83.9 & 0.838 & 4.32 \\
GBG-6 & 275 & 64 & 89.5 & 0.900 & 3.69 \\
GBG-7 & 343 & 64 & 91.5 & 0.919 & 3.09 \\
PBLG & $2 P_{\text {A }}=1176$ & & 100.0 & 1.000 & 0 \\
\hline
\end{tabular}


temperature was controlled within $0.1{ }^{\circ} \mathrm{C}$. The measurements were carried out at temperatures between 25 and $60^{\circ} \mathrm{C}$, and a pressure between 1 and 4 atm, using compressed nitrogen gas.

\section{RESULTS AND DISCUSSION}

\section{Dynamic Mechanical Spectroscopy}

Figure 1 illustrates the temperature dependence of the loss tangent $\tan \delta$, and the storage modulus $E^{\prime}$ for GBG-4 block copolymer and poly $\gamma$-benzyl L-glutamate) [PBLG] homopolymer membranes both cast from chloroform. The loss tangent spectra of both samples show similar tendencies and a large $\beta$-peak at $40^{\circ} \mathrm{C}(110 \mathrm{~Hz})$. The storage modulus curves of these two samples are also similar and show a fairly large modulus drop at the $\beta$-peak. Such behavior, referred to as the $\beta$-process, was reported with the PBLG homopolymer, ${ }^{4}$ and is strongly affected by the crystalline contribution of the sample ${ }^{5}$ Differential scanning calorimetry ${ }^{6}$ and dielectric relaxation ${ }^{7}$ studies indicated that the $\beta$-process more

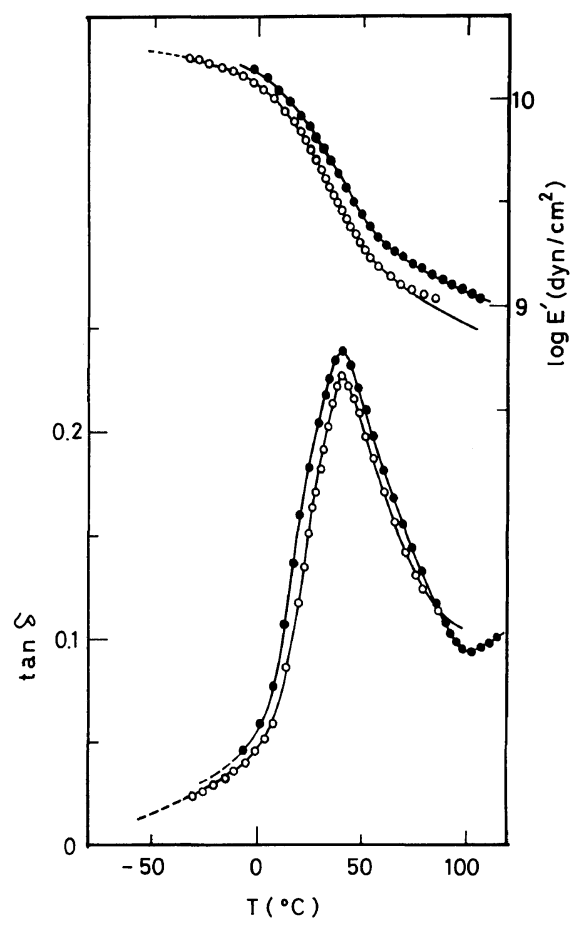

Figure 1. Temperature dependences of $\tan \delta$ and $\log E^{\prime}$ for GBG-4 (O) block copolymer and PBLG ( $\odot)$ homopolymer membranes cast from chloroform. closely resembled the glass-rubber transition. A consistent hypothesis is that the side chains of polypeptide molecules do not crystallize in the solid state but consitute relatively disordered phase filling the lattice space between ordered, helical backbone chains. As is obvious in Figure 1, the peak profile of the GBG block copolymer resembles that of the PBLG homopolymer; the peak heights, however, are different since polybutadiene is viscoelastically inactive over the temperature range investigated.

Takayanagi $^{3}$ has succeeded in representing the viscoelastic properties of heterogeneous polymer systems in terms of a simple mechanical model comprised of elements connected partly in series and partly in parallel. The individual elements are assumed to have the viscoelastic properties of the constituent phases. Two parameters, $\lambda$ and $\psi$, which express the mixing state of the system, were introduced in his model. ${ }^{3}$ The relative magnitude of $\lambda$ to $\psi$ can be interpreted as representing the extent of the parallel to the series character of the observed behavior, and $\lambda \psi$ is equal to the volume fraction of the inclusion phase; i.e., $\varphi=\lambda \psi$. The dynamic Young's modulus $E_{\mathrm{c}}{ }^{*}$ for such a model is given by

$$
\frac{1}{E_{\mathrm{c}}^{*}}=\frac{\psi}{\lambda E_{2}^{*}+(1-\lambda) E_{1}^{*}}+\frac{1-\psi}{E_{1}^{*}}
$$

if an assumption is made that the elastic Poisson's ratio $v$ is $v=v_{1}=v_{2}=0.5$ (the subscripts 1 and 2 refer to matrix and inclusion phase, respectively). Equation 1 is equivalent to the modified Kerner's equation used by Dickie. ${ }^{8}$ The expression for $E_{\mathrm{c}}{ }^{*}$ can be separated into real and imaginary parts to give explicit expressions for the dynamic elastic modulus $E^{\prime}$ and the loss modulus $E^{\prime \prime}$.

$$
\begin{aligned}
E^{\prime} & =\left|E_{\mathrm{c}}{ }^{*}\right| \cos \delta \\
E^{\prime \prime} & =\left|E_{\mathrm{c}}{ }^{*}\right| \sin \delta
\end{aligned}
$$

Equation 1 is applicable to the block copolymer systems investigated in this work, since the G-block of GBG block copolymers takes the same conformation as that of homopolypeptide. ${ }^{2}$

In Figure 2, the dynamic modulus $E^{\prime}$ and the loss modulus $E^{\prime \prime}$ calculated by using eq 1,2 , and 3 were compared with observed data for GBG-4 block copolymer membrane. Broken curves in Figure 2 are the experimental results for PBLG homopolymer. Since no information is available for the dynamic 


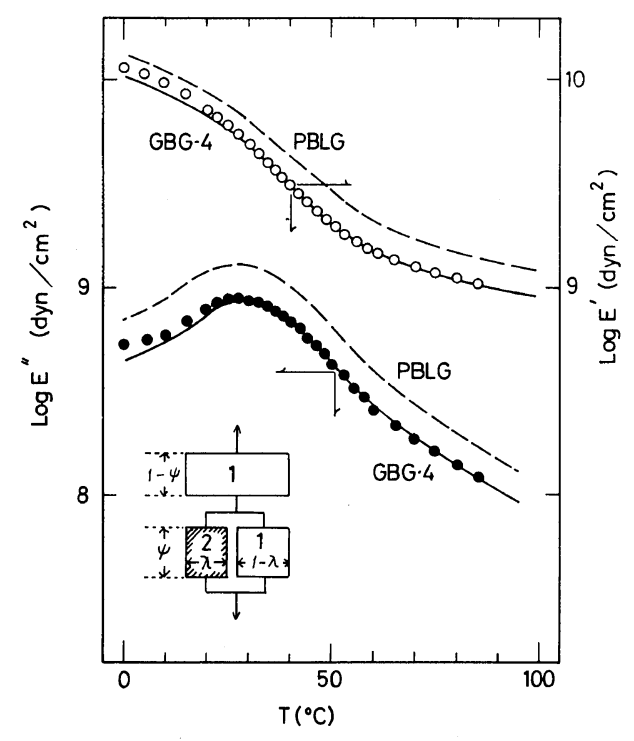

Figure 2. Temperature dependences of $\log E^{\prime}$ and $\log$ $E^{\prime \prime}$ for GBG-4 block copolymer membrane cast from chloroform. The solid curves are calculated from eq 1 with values of $\lambda=0.56, \psi=0.34$, and $\varphi=0.19$. The broken curves denote the experimental results on PBLG homopolymer membrane.

modulus $E^{\prime}$ of polybutadiene for the temperature range investigated here, $E^{\prime}=5 \times 10^{7} \mathrm{dyn}^{-2}$ was used according to Takayanagi et al. ${ }^{9}$ In the present system, the polybutadiene component is considered to be dispersed in the matrix phase of poly $(\gamma$-benzyl L-glutamate) component in the GBG block copolymer membrane as indicated by the electron microscopy. ${ }^{2}$ For this calculation, the volume fraction $\varphi$ of polybutadiene component in the membrane was estimated as 0.19 from the result of molecular characterization, assuming that the polybutadiene chains in the solid membrane are of unperturbed chain dimension. The solid curves in Figure 2 are obtained by using $\varphi=0.19, \lambda=0.56$, and $\psi=0.34$, the latter two values were determined by the trial-and error method based on the experimental data. If $\lambda$ value larger than 0.56 is adopted, the calculated value of $E^{\prime}$, as well as $E^{\prime \prime}$, will be smaller than the calculated value obtained above, and if $\lambda$ is decreased, the calculated value of $E^{\prime}$ is increased. As is obvious from Figure 2, a rather satisfactory agreement was obtained between the observed and calculated curves, except for some deviation in the lower temperature region, on the basis of a reasonable value of $\varphi$.
If the shape of inclusion phase is sphere, then the values of $\lambda$ and $\psi$ are given by ${ }^{10}$

$$
\begin{aligned}
& \lambda=\frac{2+3 \varphi}{5} \\
& \psi=\frac{5 \varphi}{2+3 \varphi}
\end{aligned}
$$

When we adopt $\varphi=0.19$ in eq 4 , we obtain $\lambda=0.51$. The best-fit $\lambda$-value 0.56 estimated for the GBG-4 block copolymer is somewhat larger than the value for spherical inclusion, suggesting that the amount of the lines of force in the membrane passing through the matrix component is smaller than that for spherical inclusion model. One of the possible explanations for this trend is that the shape of the inclusion phase deviates from the sphere; in other words, the ellipsoidal or cylindrical inclusion phase (domain) is expected for the polybutadiene component in this block copolymer membrane. Also, such an estimation is quite reasonable on the basis of our previous observations by electron microscopy. ${ }^{2}$

\section{Tensile Properties}

The tensile properties of block copolymer membranes are dependent on the copolymer composition, molecular weights of the blocks, and microphase structure. In general, the block copolymers of the types $\mathrm{A}-\mathrm{B}-\mathrm{A}$ and $\mathrm{A}-\mathrm{B}-\mathrm{A}-\mathrm{B}$, in which $\mathrm{A}$ and $\mathrm{B}$ denote, respectively, hard block and soft block, are quite strong, whereas types $\mathrm{A}-\mathrm{B}$ and $\mathrm{B}-\mathrm{A}-\mathrm{B}$ are brittle despite the presence of the rubbery $\mathrm{B}$ component. The tensile stress-strain parameters of the GBG block copolymers and PBLG homopolymer membranes are summarized in Table II. Young's modulus $E$ at an elongation of $1 \%$, and the strength $\sigma_{\mathrm{B}}$ at the breaking point of GBG block copolymer membranes are generally smaller than those for the PBLG homopolypeptide membrane. The values in Table II appear to depend on the extent of polybutadiene component, and decrease by increasing the content of polybutadiene block. The value of the elongation $\varepsilon_{\mathbf{B}}$ at the breaking point slightly increases with an increase in the content of polybutadiene.

\section{Permeability}

The hydraulic permeability $K$ of water is defined $^{11-14}$ by

$$
J_{\mathrm{f}}=K(\Delta P / \Delta x)
$$


Table II. Stress-strain parameters for membranes of GBG block copolymers and PBLG homopolymer

\begin{tabular}{ccccr}
\hline Designation & Butadiene $/ \mathrm{mol}^{\circ} \%$ & $E / \mathrm{dyn} \mathrm{cm}^{-2}$ & $\sigma_{\mathrm{B}} / \mathrm{dyn} \mathrm{cm}^{-2}$ & $\varepsilon_{\mathrm{B}} / \%$ \\
\hline GBG-1 & 32.5 & $1.78 \times 10^{9}$ & $0.42 \times 10^{8}$ & 98 \\
GBG-3 & 21.9 & $3.08 \times 10^{9}$ & $0.78 \times 10^{8}$ & 130 \\
GBG-5 & 16.1 & $3.84 \times 10^{9}$ & $1.14 \times 10^{8}$ & 95 \\
GBG-6 & 10.5 & $4.28 \times 10^{9}$ & $1.50 \times 10^{8}$ & 80 \\
GBG-7 & 8.5 & $4.77 \times 10^{9}$ & $1.89 \times 10^{8}$ & 94 \\
PBLG & 0.0 & $5.69 \times 10^{9}$ & $2.23 \times 10^{8}$ & 94 \\
\hline
\end{tabular}

where $J_{f}$ is the flux of water per unit area of membrane subjected to a hydraulic pressure difference $\Delta P$ across the membrane whose thickness is $\Delta x$.

Figure 3 illustrates the relation between the water flux $J_{\mathrm{f}}\left(\mathrm{cm} \mathrm{s}^{-1}\right)$ and the applied pressure $\Delta P(\mathrm{~atm})$ at various temperatures for GBG-6 block copolymer membrane (31 $\mu \mathrm{m}$ in thickness) cast from chloroform. In the pressure range examined, the relation $J_{\mathrm{f}}$ $v s . \Delta P$ is linear, indicating that the effect of compression on the membrane is negligible. The water flux increases with increasing temperature and applied pressure. The experimental results suggest that the GBG block copolymer membrane is a uniform matrix in the direction of the membrane thickness. From Figure 3, the $K$-value at each temperature was calculated by using eq 6 . As shown in Table III, the $K$ value was independent of the membrane thickness investigated.

Figure 4 shows the temperature dependence of the hydraulic permeability $K$ of water for GBG block

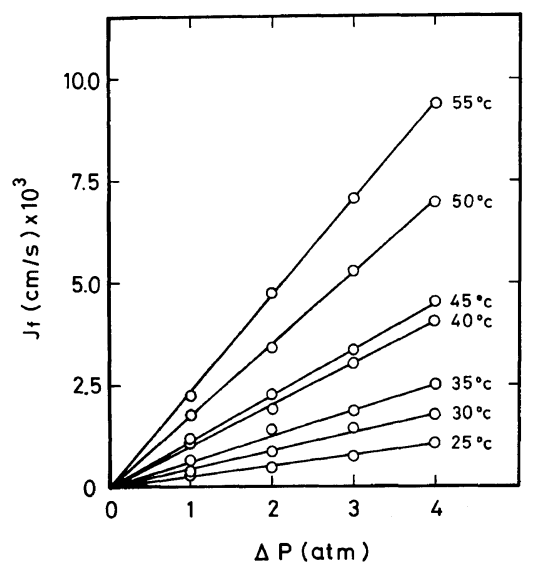

Figure 3. Water flux $J_{\mathrm{f}}$ plotted against pressure $\Delta P$ at various temperatures for GBG-6 block copolymer membrane cast from chloroform ( $31 \mu \mathrm{m}$ in thickness).
Table III. Hydraulic permeability $K$ obtained with GBG-4 block copolymer membranes of different thickness

\begin{tabular}{crc}
\hline$\Delta x / \mathrm{cm}$ & $J_{\mathrm{f}} / \mathrm{cm} \mathrm{s}^{-1}$ & $K / \mathrm{cm}^{2} \mathrm{~s}^{-1} \mathrm{~atm}^{-1}$ \\
\hline $24 \times 10^{-4}$ & $12.26 \times 10^{-4}$ & $1.01 \times 10^{-6}$ \\
$33 \times 10^{-4}$ & $8.89 \times 10^{-4}$ & $1.01 \times 10^{-6}$ \\
$38 \times 10^{-4}$ & $7.88 \times 10^{-4}$ & $1.03 \times 10^{-6}$ \\
\hline
\end{tabular}

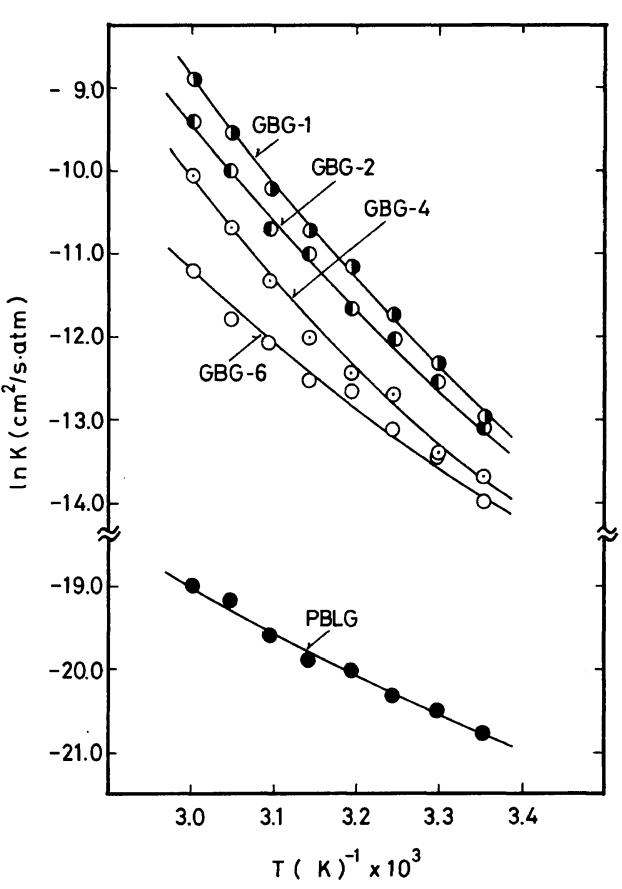

Figure 4. Temperature dependences of hydraulic permeability $K$ of water for GBG block copolymer membranes and PBLG homopolymer membrane cast from chloroform. 


\section{A. Nakajima, K. Kugo, and T. Hayashi}

copolymers and PBLG homopolymer membranes. The $K$-values at $25^{\circ} \mathrm{C}$ are given in Table IV together with the values of activation energy $E_{\mathrm{a}}$ of hydraulic permeability calculated from the slope of the curves at $25^{\circ} \mathrm{C}$ (Figure 4). The numerical values of activation energy shown in the table are larger than the activation energy of $4 \mathrm{kcal} \mathrm{mol}^{-1}$ (at $25^{\circ} \mathrm{C}$ ) for viscous flow of water. ${ }^{15}$ Thus the water flow in the membrane is considered to be different from a purely viscous flow. The nature of the micro-heterophase structure in the membranes should play an important role for the water permeability through the membrane.

The most remarkable characteristic of the GBG block copolymer membranes compared to PBLG homopolymer membrane is that the values of the hydraulic permeability for the GBG membranes are remarkably higher than that of PBLG membrane; the $K$-value for GBG-1 is about 2000 folds of $K$-value for PBLG membrane. Such a drastic increase in the
Table IV. Hydraulic permeability $K$ and activation energy $E_{\mathrm{a}}$ for membranes of GBG block copolymers and of PBLG hompolymer at $25^{\circ} \mathrm{C}$

\begin{tabular}{|c|c|c|c|}
\hline \multirow{2}{*}{$\begin{array}{l}\text { Disigna- } \\
\text { tion }\end{array}$} & Butadiene & $K$ & $E_{\mathrm{a}}$ \\
\hline & $\mathrm{mol} \%$ & $\mathrm{~cm}^{2} \mathrm{~s}^{-1} \mathrm{~atm}^{-1}$ & $\mathrm{kcal} \mathrm{mol}^{-1}$ \\
\hline PBLG & 0.0 & $1.11 \times 10^{-9}$ & 9.7 \\
\hline GBG-6 & 10.5 & $8.65 \times 10^{-7}$ & 15.6 \\
\hline GBG-4 & 18.7 & $1.01 \times 10^{-6}$ & 18.1 \\
\hline GBG-2 & 28.4 & $2.03 \times 10^{-6}$ & 19.9 \\
\hline GBG-1 & 32.5 & $2.32 \times 10^{-6}$ & 22.5 \\
\hline
\end{tabular}

$K$-value for the GBG membrane should be attributed mainly to the specific feature of the interfacial zone between $\mathrm{G}$ and $\mathrm{B}$ domains in the GBG membranes.

Figure 5 shows a schematic representation of hydrogen-bonded water and water clusters not bonded to polymer chains in the interfacial area of

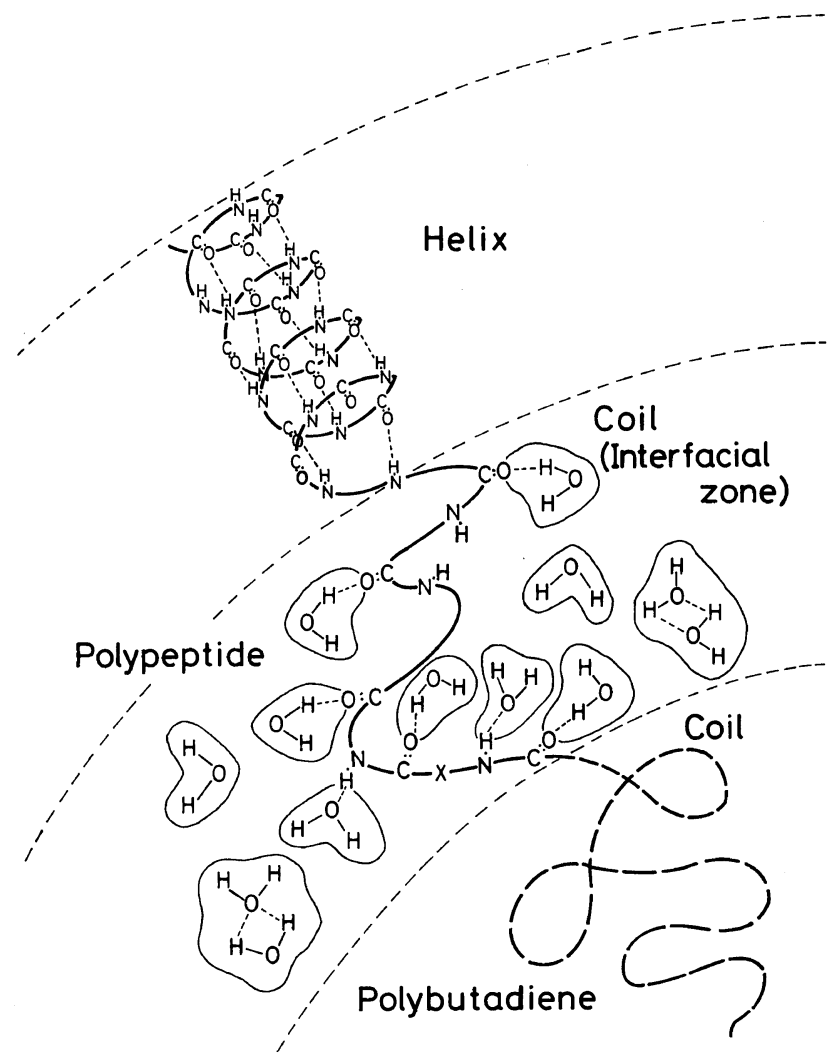

Figure 5. Schematic representation of hydrogen-bonded water and clusters of water in the interfacial zone in micro-heterophase structure of GBG block copolymer membrane. 


\section{A-B-A Blockpolymers Containing PBLG}

micro-heterophase structure of GBG block copolymer membranes. As described above, the interfacial zone is made up of the coiled peptide residues near the end of the polypeptide chain and the terminal residues of amine-terminated polybutadiene. The $\mathrm{NH}$ and $\mathrm{CO}$ residues in this region are not incorporated in intramolecular hydrogen bondings of $\alpha$-helix of polypeptide backbone, but can bind water through hydrogen bonds. Thus, the amount of the bonded water molecules should be related closely to the volume of the interfacial zone. Furthermore, such bonded water may contribute to reducing the size of the water clusters. These factors may result in the dramatically large $K$-values for GBG membranes.

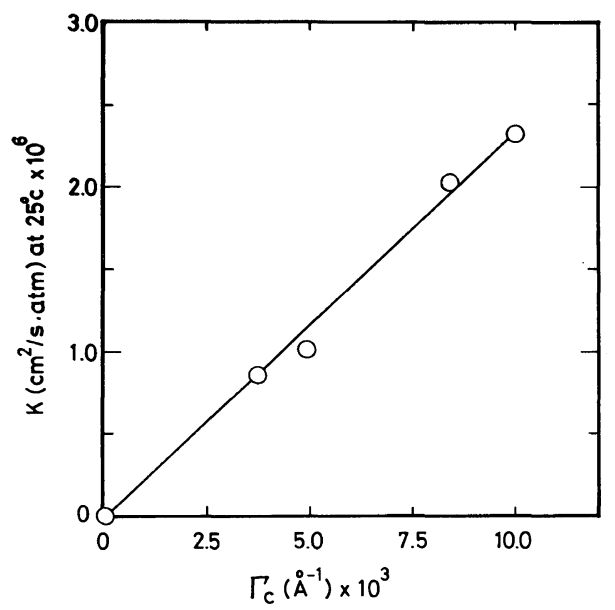

Figure 6. Hydraulic permeability $K$ of water plotted against the interfacial area $\Gamma_{\mathrm{c}}$ per unit volume for GBG block copolymer membranes of different B contents.

Finally, to intensify the contribution of the interfacial region toward water permeability, the $K$ - values were plotted against the interfacial area $\Gamma_{\mathrm{c}}$ per unit volume in Figure 6 , in which $\Gamma_{\mathrm{c}}$ is the quantity defined in the preceding paper. ${ }^{2}$ It was shown that $K$ increases in proportion to $\Gamma_{\mathrm{c}}$. This result should support our conclusion that the interfacial region is responsible for the water permeability and the hydraulic permeability of water increases with increasing polybutadiene content of the GBG block copolymers.

\section{REFERENCES}

1. A. Nakajima, T. Hayashi, K. Kugo, and K. Shinoda, Macromolecules, 12, 000 (1979).

2. A. Nakajima, K. Kugo, and T. Hayashi, Macromolecules, 12, 000 (1979)

3. M. Takayanagi, Memoir of Faculty of Engineering, Kyushu University, 23, 41 (1963).

4. T. Hayashi, J. M. Anderson, and A. Hiltner, Macromolecules, 10, 352 (1977).

5. T. Fukuzawa, I. Uematsu, and Y. Uematsu, Polym. J., 6, 431 (1974).

6. G. Peggion, G. Ceccorulli, M. Pizzoli, and E. Peggion, Macromolecules, 8, 762 (1975).

7. A. Tsutsumi, K. Hikichi, T. Takahashi, Y. Yamashita, N. Matsushima, M. Kanbe, and M. Kaneko, J. Macromol. Sci., Phys., 8, 413.(1973).

8. R. A. Dickie, J. Appl. Polym. Sci., 17, 45 (1973).

9. S. Uemura and M. Takayanagi, J. Appl. Polym. Sci., 10, 113 (1966).

10. M. Takayanagi, S. Uemura, and S. Minami, J. Polym. Sci., C5, 113 (1965).

11. H. Yasuda, C. E. Lamaze, and A. Peterlin, J. Polym. Sci., A-2, 9, 1117 (1971).

12. H. Yasuda and C. E. Lamaze, J. Polym. Sci., A-2, 9, 1537 (1971).

13. H. Yasuda, C. E. Lamaze, and A. Schindler, J. Polym. Sci., $A-2$, 9, 1579 (1971).

14. A. Peterlin, H. Yasuda, and H. G. Olf, J. Appl. Polym. Sci., 16, 865 (1972).

15. R. A. Horne, "Water and Aqueous Solutions," Wiley, New York, N.Y., 1972, p 704. 